\title{
Fabrication and simulation of nanostructures for domain wall magnetoresistance studies on Nickel
}

\author{
D. Claudio-Gonzalez ${ }^{\mathrm{a}, *}$, M. K. Husain ${ }^{\mathrm{a}}$, C. H. de Groot $^{\mathrm{a}}$, G. Bordignon ${ }^{\mathrm{b}}$, T. Fischbacher ${ }^{\mathrm{b}}$, H. Fangohr ${ }^{\mathrm{b}}$ \\ ${ }^{\mathrm{a}}$ School of Electronics and Computer Science, University of Southampton, Southampton SO17 1BJ, United Kingdom \\ ${ }^{\mathrm{b}}$ School of Engineering Sciences, University of Southampton, Southampton SO17 1BJ, United Kingdom
}

\section{A R T I C L E I N F O}

\section{Article history:}

Received 12 June 2005

Accepted 14 June 2005

Online publication

\section{PACS:}

81.07.-b;

85.75.-d;

73.63.-b

\section{Keywords:}

domain wall; mag-

netoresistance;

dwmr; constrained

geometries

\begin{abstract}
A B S T R A C T
We report the use of electron beam lithography and a bilayer liftoff process to fabricate magnetic Ni nanostructures with constriction widths in the range of 22 to $41 \mathrm{~nm}$. The structures fabricated correspond to the nanobridge geometry. Reproducibility and control over the final nanostructure geometry were observed when using the fabrication process introduced, these two qualities are important in order to carry out a more systematic analysis of domain wall magnetoresistance (DWMR). On the other hand, micromagnetic simulations of structures with the nanobridge geometry were carried out using not only the dimensions of the fabricated nanostructures but also smaller dimensions thought to be achievable with further optimization of the fabrication process. It was found that domain walls with a reduced length of $42.5 \mathrm{~nm}$ can be obtained using the nanobridge geometry. Furthermore, the anisotropic magnetoresistance (AMR) effect was calculated numerically and it was found to be smaller than the DWMR, this makes the nanobridge geometry a good candidate for future measurements of the magnetoresistive effect due to domain wall scattering.
\end{abstract}

\section{Introduction}

The importance of studying magnetic domain walls (DWs) is due to the variety of physical phenomena arising from their interaction with magnetic fields and electric currents. As shown by Bruno [1], DWs can be tailored by defining constrictions in a ferromagnetic structure. Garcia et al. [2] reported large values of magnetoresistance which were attributed to domain wall scattering due to the inability of electron spin to travel across the DW adiabatically when its length is comparable to the Fermi wavelength [3]. This opened the possibility of using DWs as the source for a large magnetoresistance effect. Experiments on domain wall magnetoresistance (DWMR) showing a positive effect have been reported using different ferromagnetic structures such as thin films [4], cross-shaped junctions [5], zigzag wires [6], constricted wires $[7,8]$, and micrometer-sized elements [9]. Micromagnetic simulation studies of constricted wires and nanobridges have already carried out $[10,11]$. In the latter, we reported the possibility to reduce the DW length down to $11 \mathrm{~nm}$ using a nanobridge geometry when taking into account a weak in-plane anisotropy.

In this study, the process for the fabrication of nanostructures with the nanobridge geometry is presented. Furthermore, micromagnetic simulation studies were performed to estimate the width of the DWs on the fabricated structures and the results are presented. The AMR effect for the nanobridge geometry is calculated numerically using the actual dimensions observed in the fabricated structures. Since our aim is to carry out experimental measurements of DWMR on nanobridges

\footnotetext{
* Corresponding author.

Email address: dcg04r@ecs.soton.ac.uk (D. Claudio-Gonzalez).
}

in future work, the simulation of the AMR was done to assess whether AMR was higher than DWMR in these structures. This is accomplished by comparing the AMR effect, as obtained from simulations, to the analytical prediction of the DWMR effect. These results suggest that future magnetoresistance measurements will reflect the scattering due to the presence of a DW in the nanobridge constriction.

\section{Experimental}

For the fabrication of the nanostructures presented in this paper we used Si n-type $<100>$ wafers with resistivity $17-33 \Omega \cdot \mathrm{cm}$ as a substrate. A 20-nm-thick layer of $\mathrm{SiO}_{2}$ was then thermally grown on the front side of the wafer. The adhesive agent hexamethyldisilazane (HDMS) was used as a primer layer before the spinning of a 250-nm-thick layer of anisole-based resist ZEP520-A. Patterning was done with electron beam lithography (EBL) using a JEOL Electron Beam Pattern Generator (JBX-9300FS) at $100 \mathrm{keV}$ with a dose of $115 \mu \mathrm{C} / \mathrm{cm}^{2}$. Development of the exposed wafer was done using a MIBK:IPA process. Subsequently, the wafer was wet etched with buffered hydrofluoric acid BHF (7:1) for $42 \mathrm{sec}$ in order to create an undercut in the $\mathrm{SiO}_{2}$ layer. Electron-gun evaporation (at a pressure of $10^{-7}$ mbar) was used to deposit a $10-\mathrm{nm}$-thick layer of $\mathrm{Ni}$ and a 1nm-thick Au capping layer without breaking the vacuum. Liftoff was carried out in acetone on a wobbler.

The nanobridge geometry consists of a constriction connecting two rectangular pads as shown in Figures 1a and 1b. Left and right pads are 300-nm-wide with lengths of 100 , and $200 \mathrm{~nm}$ respectively. This difference in the pad lengths is proposed to facilitate a magnetic configuration in which a domain wall is formed at the constriction due 


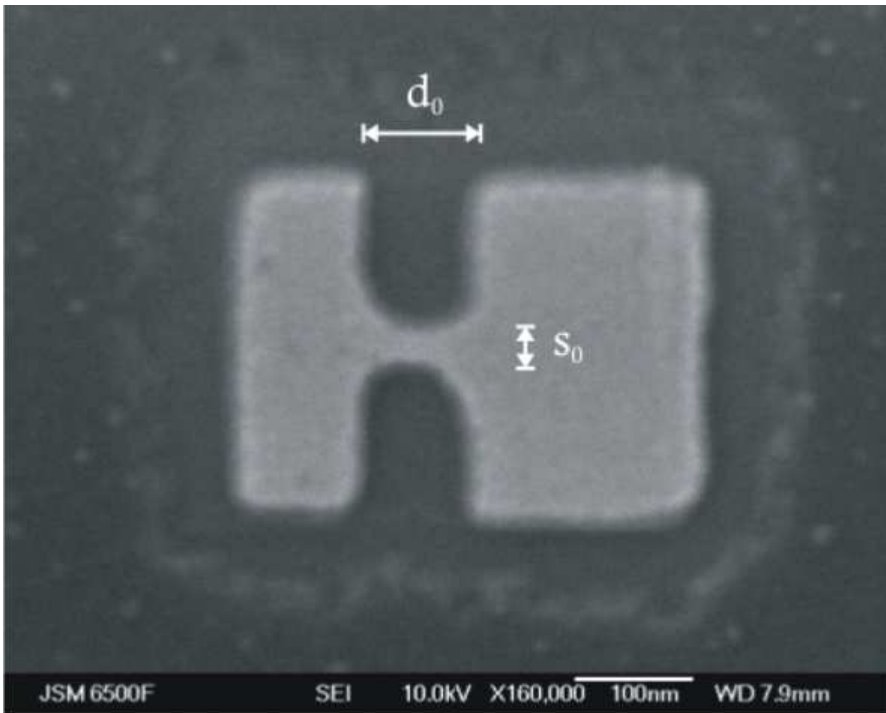

(a)

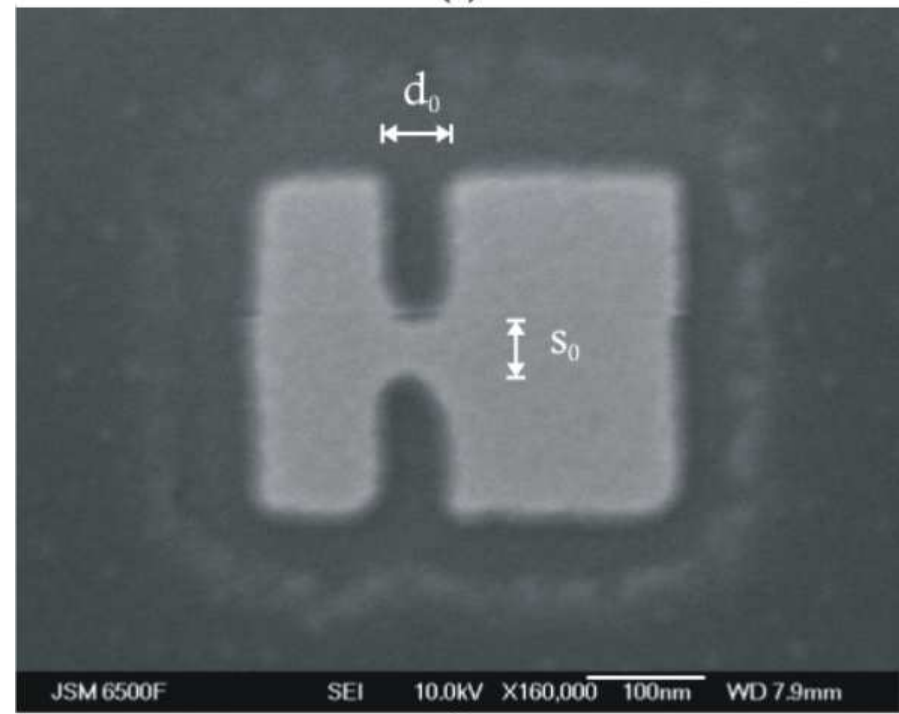

(b)

Fig. 1. SEM micrographs of nanostructures with a nanobridge geometry. Nominal lengths of the constrictions were $d_{0}=100 \mathrm{~nm}(\mathrm{a})$, and $\mathrm{d}_{0}=60 \mathrm{~nm}(\mathrm{~b})$. Nominal width for both structures was $\mathrm{s}_{0}=20 \mathrm{~nm}$. Although the obtained constriction lengths are virtually the same as the nominal values, the observed widths are: $\mathrm{s}_{0}=22$ for the case of (a), and $41 \mathrm{~nm}$ for (b)

to the different coercivities at which the magnetization in the pads switches.

To study the magnetic behaviour of the fabricated structures, we used the micromagnetics code based on finite difference method OOMMF [12]. Common parameters for $\mathrm{Ni}$ were used: $\mathrm{M}_{s}=490 \mathrm{kA} / \mathrm{m}$, and A $=9 \times 10^{-12} \mathrm{~J} / \mathrm{m}$. Where $\mathrm{M}_{s}$ is the saturation magnetization, and $\mathrm{A}$ is the exchange stiffness. The cell size was $5 \mathrm{~nm}^{3}$. The magnetization reversal of the nanobridges for fields from +100 to $-100 \mathrm{mT}$ with 5 $\mathrm{mT}$ steps applied along the $y$ axis (as displayed in Figure 1), were simulated using the aforementioned parameters.

Magnetization distributions of the nanobridge at fields where a DW is pinned in the constriction were used to estimate the width of the DW. The DW profiles obtained from such simulations are shown in Figure 2 along with their respective widths which were calculated

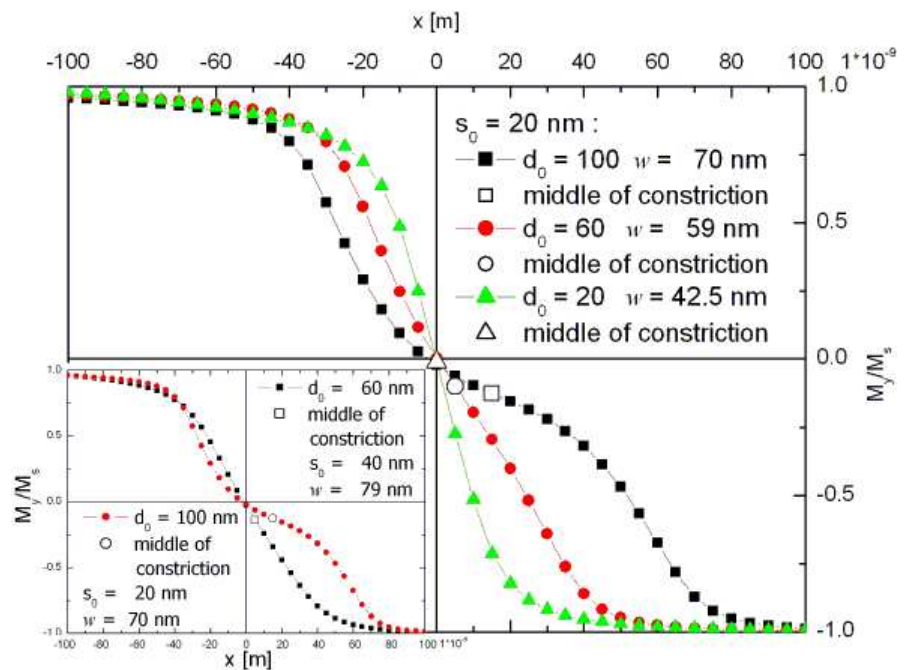

Fig. 2. Simulated domain wall profiles in nanobridges as a function of the constriction length. From micromagnetic simulations it was found that a domain wall length of $w \approx 42.5 \mathrm{~nm}$, is achievable with the nanobridge geometry (Definition of the DW length is the same as the one used in Ref. [11]). This domain wall length corresponds to a constriction of dimensions $\mathrm{s}_{0}=\mathrm{d}_{0}=20 \mathrm{~nm}$. In the inset the simulated domain profiles corresponding to the fabricated nanobridges illustrated in Figures $1 \mathrm{a}$ and $1 \mathrm{~b}$ are shown. In all cases the position of the DW can be realized by examining the geometric middle point of the constriction indicated for each profile. As the constriction length decreases, the DW is pushed towards the middle of the constriction.

using the method introduced in our previous work on nanobridges [11]. Simulated DW lengths for the nanostructures featured in Figure 1, are displayed as an inset in Figure 2. For the calculation of the anisotropic magnetoresistance (AMR) plotted in Figure 3b we used nmag (a multiphysics package based on the finite element method developed at Southampton University [13]) and followed the methodology employed by Bordignon et al. in Reference [14]. The advantage of using this method is, that it takes into account the back reaction of the AMR effect onto the current distribution hence it provides a more accurate AMR estimation. The plots shown in Figures 3a and 3b correspond to the magnetization reversal and the AMR numerically calculated of a nanobridge with the dimensions of the structure depicted in Figure 1a. This calculation was carried out using an experimental resistivity $\left(\rho_{0}=52 \Omega \cdot \mathrm{cm}\right)$ obtained from I-V measurements.

In order to estimate the resistance change due to the presence of a DW in our constricted nanostructures we employed the equation proposed by Ieda et al. [15].

$\Delta R=2 P^{2} \rho_{0} \lambda_{F} A^{-1} F(\xi)$

Where $P$ is the polarization of the conduction spin, $\rho_{0}$ is the normal resistivity, $\lambda_{F}$ is the spin diffusion length, $A$ is the cross sectional area of the constriction, and $F(\xi)$ is a function of the ratio $w / \lambda_{F}$ where $w$ is the length of the DW. A spin polarization of $\mathrm{P}=20 \%$, and a spin diffusion length $\lambda_{F}=21 \mathrm{~nm}$ for $\mathrm{Ni}$ as determined by recent experiments $[17,18]$ were considered. If we add this change of resistance to the resistance measured in similar structures without an applied magnetic field $(\mathrm{R} \approx 345 \pm 0.1 \Omega$ ), we can then estimate the MR ratios presented in Table 1 . 


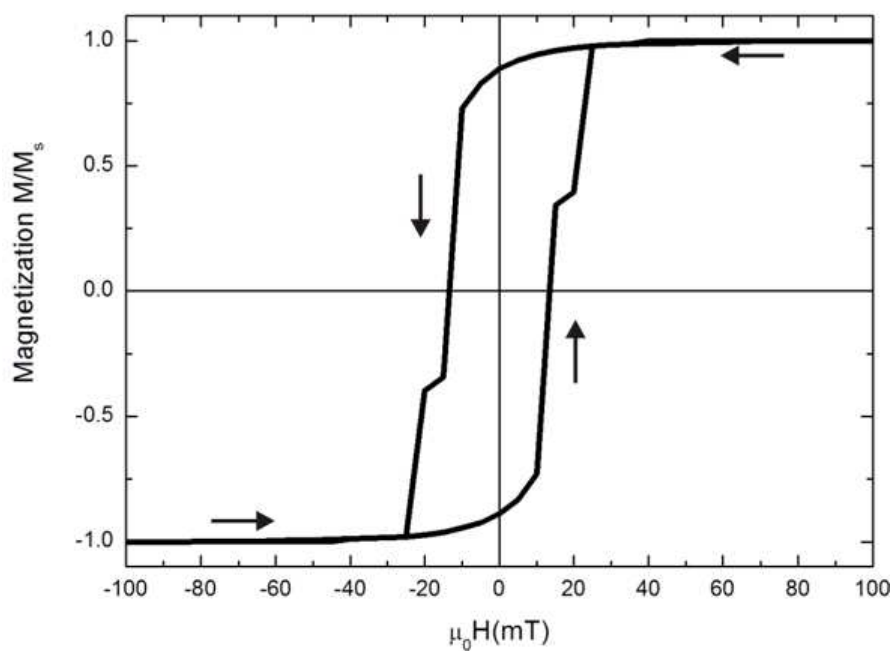

(a)

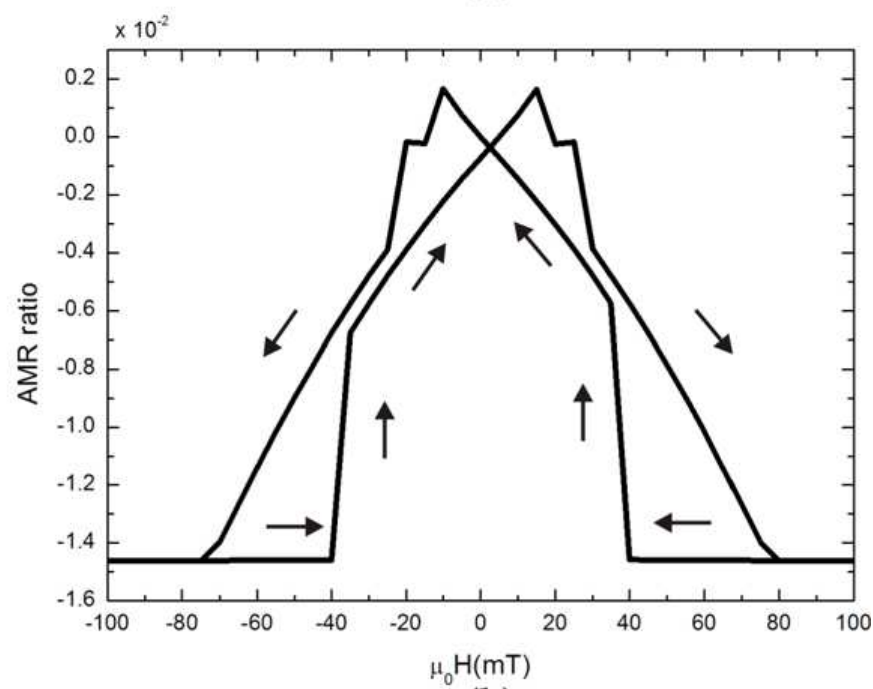

(b)

Fig. 3. (a) Magnetization reversal of a nanobridge with constriction dimensions: $\mathrm{s}_{0}=22 \mathrm{~nm}$ and $\mathrm{d}_{0}=100 \mathrm{~nm}$ for magnetic fields applied along the $y$ axis. (b) AMR effects of $4.9 \times 10^{-4}$ and $2.37 \times 10^{-3}$ are expected at -15 and $15 \mathrm{mT}$ fields. These are the fields at which a domain wall is formed at the constriction, this is represented by the steps in the magnetization reversal shown in (a).

\section{Results and discussion}

From the structures shown in Figure 1 we can observe that the nanobridge geometry is very well defined. The correlation between the actual and nominal dimensions, more remarkably over the rectangular pads, can be readily seen. This constitutes evidence that a high degree of control over the final structures is possible thanks to the fabrication process introduced above. Similar results were observed for different samples featuring the same structure, this ensures the reproducibility required in order to carry out a more systematic analysis of DWMR. From the inspection of the plot shown in Figure $3 \mathrm{a}$ one can observe steps in the magnetization reversal at $\pm 15 \mathrm{mT}$ fields. By inspecting the simulated magnetization distribution corresponding to this fields, it was found that such steps correspond to the pinning of a DW at the constriction. The profiles of the DWs are shown in the inset of
Figure 2. For a nanobridge with the dimensions showed in Figure 1a ( $\mathrm{s}_{0}=22 \mathrm{~nm}, \mathrm{~d}_{0}=100 \mathrm{~nm}$ ), the length of the DW is $w=70 \mathrm{~nm}$. For this constriction length, it is more favourable for the magnetization to split into two $90^{\circ} \mathrm{DWs}$ [11], as reflected by the profiles observed. Since our aim is to experimentally achieve smaller DW lengths, we studied the effect that further reduction of the constriction dimensions has on the resulting DW length. In order to do so, we calculated the DW width for constrictions with width $\mathrm{s}_{0}=20 \mathrm{~nm}$ and lengths $\mathrm{d}_{0}=20$, 60, and $100 \mathrm{~nm}$. Such results are shown in Figure 2. When comparing the DW widths from this plot with those of the inset it becomes evident that constrictions with smaller widths have greater influence on the DW length than constrictions with just shorter lengths. This is an important finding because, from an experimental point of view, it means that we still need to optimize our fabrication process to realize smaller constriction widths, and hence shorter DW lengths. From the results shown in Figure 2 we observe that a DW with length $w=42.5$ $\mathrm{nm}$ is expected for a constriction with dimensions $\mathrm{s}_{0}=20 \mathrm{~nm}$ and $\mathrm{d}_{0}=20 \mathrm{~nm}$. Nonetheless, a constriction with dimensions $\mathrm{s}_{0}=20$ $\mathrm{nm}$ and $\mathrm{d}_{0}=60 \mathrm{~nm}$ seems more plausible. The estimated values of DWMR using equation 1 are presented in Table 1. From these results, we observe that DWMR can be higher than AMR.

\begin{tabular}{ccccc}
\hline DW length & $\begin{array}{c}\text { Constriction } \\
\text { dimensions }\end{array}$ & $\begin{array}{c}\Delta \mathbf{R} \\
(\mathrm{Eq} 1)\end{array}$ & AMR & DWMR \\
$(w)[\mathrm{nm}]$ & $\left(\mathrm{s}_{0}[\mathrm{~nm}], \mathrm{d}_{0}[\mathrm{~nm}]\right)$ & {$[\Omega]$} & & \\
\hline 70 & 22,100 & 1.11 & $2.37 \times 10^{-3}$ & $3.5 \times 10^{-3}$ \\
79 & 41,60 & 0.6 & $1.1 \times 10^{-3}$ & $1.8 \times 10^{-3}$ \\
60 & 20,60 & 1.53 & - & $4.4 \times 10^{-3}$ \\
\hline
\end{tabular}

Table 1

Estimated DWMR as a function of the DW lengths obtained from simulations of nanobridges with different constriction lengths. A shorter DW length means a higher contribution to the MR effect.

\section{Conclusions}

We succeeded in fabricating nanostructures with constriction widths in the range of 22 to $41 \mathrm{~nm}$ using a nanobridge geometry. Domain walls with reduced widths down to $42.5 \mathrm{~nm}$ can be obtained by using this geometry. Both AMR and DWMR have been estimated using numerical and micromagnetic simulations. It was found that DWMR is higher than AMR, this makes the nanobridge geometry a good candidate to carry out further studies on the topic of domain wall magnetoresistance. Experimental work is taking place to obtain DWMR measurements on such structures. D. Claudio-Gonzalez acknowledges support from the Mexican Council of Science and Technology (CONACYT). We also thank Mir Mokthari from Mir Enterprises Limited and Enrico Gili for their contribution to the fabrication of the experimental samples.

\section{References}

[1] P. Bruno, Phys. Rev. Lett. 83 (1999) 2425.

[2] N. Garcia, M. Munoz, and Y.W. Zhao, Phys. Rev. Lett. 82 (1999) 2923.

[3] G. Tatara, Y.W. Zhao, M. Munoz, and N. Garcia, Phys. Rev. Lett. 83 (1999) 2030.

[4] M. Viret, D. Vignoles, D. Cole, J. M. D. Coey, W. Allen, D. S. Daniel, and J. F. Gregg, Phys, Rev. B 53 (1996) 8464.

[5] Y. B. Xu, C. A. F. Vaz, A. Hirohata, H. T. Leung, C. C. Yao, J. A. C. Bland, E. Cambril, F. Rousseaux, and H. Launois, Phys. Rev. B. 61 (2000) 14901 
[6] J. L. Tsai, S.F. Lee, Y. D. Yao, C. Yu, and S. H. Liou, J. Appl. Phys. 91 (2002) 7983

[7] S. Lepadatu, and Y. B. Xu, Phys. Rev. Lett. 92 (2004) 127201. S. Lepadatu, and Y. B. Xu, IEEE Trans. Magn. 40 (2004) 2688

[8] N. Garcia, C. Hao, L. Yonghya, M. Munoz, Y. Chen, Z. Cui, Z. Lu, G. Pan, A. A. Pasa. Appl. Phys. Lett. 89 (2006) 083112.

[9] P. Jubert, R. Allenspach, and A. Bischof, Phys. Rev. B 69 (2004) 220410(R)

[10] G. D. Li, Y. Zhai, P. K. J. Wong, D. X. Niu, Y. X. Lu, S. Lepadatu, and Y. B. Xu, IEEE Trans. Magn. 43 (2007) 2830

[11] H. Fangohr, J. R. Zimmermann, R. P. Boardman, D. C. Gonzalez, and C. H. de Groot J. Appl. Phys. 103 (2008) 07 D926

[12] M. Donahue and D. Porter, Object Oriented Micro-Magnetic Framework (OOMMF), www.nist.gov/oommf.

[13] T. Fischbacher, M. Franchin, G. Bordignon, and H. Fangohr. A Systematic Approach to Multiphysics Extensions of Finite-Element- Based Micromagnetic Simulations: Nmag, IEEE Transactions on Magnetics, 43, 6, 2896-2898 (2007), http://nmag.soton.ac.uk

[14] G. Bordignon, T. Fischbacher, M. Franchin, J.P. Zimmermann, A.A. Zhukov, V.V. Metlushko, P.A.J de Groot, and H. Fangohr. IEEE Trans. Magn. 43 (2007) 2881.

[15] J. Ieda, S. Takahashi, M. Ichimura, H. Imamura, and S. Maekawa. J. Magn. Mag. Mat. 310 (2007) 2058-2060

[16] H. Zare-Kolsaraki, and H. Micklitz. J. Magn. Mag. Mat. 296 (2006) 9-12

[17] C. E. Moreau, I. C. Moraru, N. O. Birge, W. P. Pratt Jr. App. Phys. Lett. $90(2007) 012101$ 